\title{
Tadeusz BUCHALCZYK
}

\author{
EINSEITIGE GEBISSANOMALIE \\ BEI NEOMYS FODIENS PEN N A N T 1771 \\ JEDNOSTRONNA ANOMALIA UZEBBIENIA \\ U NEOMYS FODIENS PENNANT 1771
}

Gebissanomalien sind bei Soricidae bekannt: Z. B. deutliche Zweikegeligkeit bei typischen einspitzigen Kegelzähnen, Fehlen von einzelnen Zähnen ( $\mathrm{J}$ a ckson, 1928; Schaefer, 1935; B u chalczyk, 1958), oder Auftreten eines überzähligen Zahnes (S e t z er, 1957).

In den Sammlungen von im Areal des Białowieżaer Nationalparkes gefangenen Kleinsäugern fand man ein Exemplar von Neomys fodiens $\mathrm{P}$ e $\mathrm{n} \mathrm{n}$ a $\mathrm{n}$ 1771 vor, das eine deutliche Gebissanomalie aufgewiesen hatte. Dieses Individuum ( $\mathrm{Nr}$ coll. 7396 leg. I. B. L. o juv.) hat eine Cb-Länge des Schädels von $21,5 \mathrm{~mm}$. In der linken oberen Zahnreihe ist $\mathrm{I}^{3}$ mit $\mathrm{C}^{1}$ in einen zweikegeligen Zahn verwachsen, dessen Grösse in Annäherung $\mathrm{I}^{2}$ gleich ist. (Phot 1). Im Vergleich mit der normalen Zahnreihe des rechten Kiefers ist im verwachsenen Zahn der Kegel des Eckzahnes stark nach vorn verschoben. Er

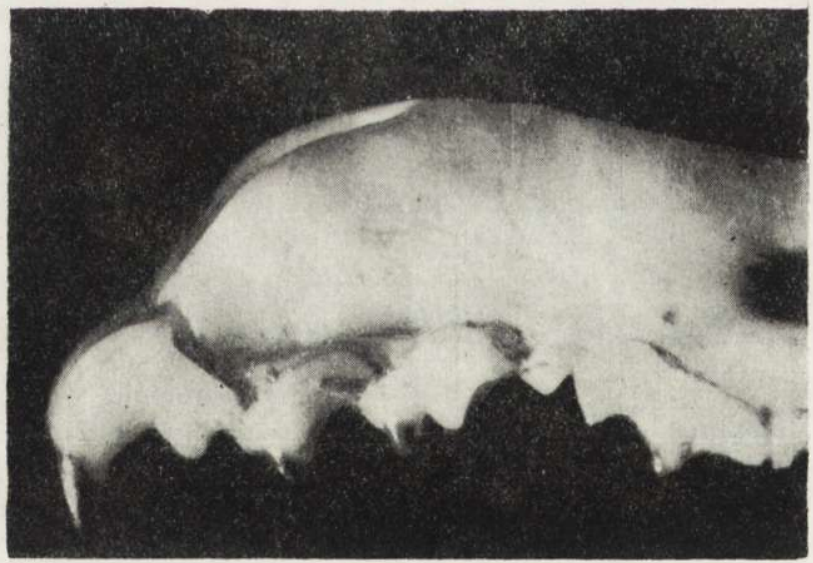

kontaktiert mit den benachbarten Zähnen nicht so genau wie der entsprechende Zahn im rechten Kiefer. Die anormale Bezahnung des Kiefers beeinflusst auf keine sichtliche Weise die Zahnanordnung der ihr entsprechenden Kieferhälfte. Sie verursacht ebenfalls keinen Unterschied in der Gesamtlänge der oberen Zahnreihen, die für beide Seiten $10,3 \mathrm{~mm}$ beträgt.

Solch ein Typ einer Gebissanomalie ist mit dem von Jones (1957) bei Crocidura lasiura Dobson 1890 beschriebenen Fall analog. Er beruht auf dem Verwachsen der Kronen von zwei benachbarten Zähnen, bei Beibehaltung von aparten 'Wurzeln in den eigenen Alveolen.

\section{SCHRIFTTUM}

Buchalczyk, T. - Die Feldspitzmaus - Crocidura leucodon (H e r$\mathrm{m} a \mathrm{n} n$ ) in den nordöstlichen Gebieten Polens. Acta Theriol., 3: 55-70. Białowieża, 1958. J a cks o n, H. H. T. - A taxonomic review of the american 
Long-tailed Shrews. N. Amer. Fauna, 51: 1-30, 1928. Jones, J. K. Jr. A dental abnormality in the shrew, Crocidura lasiura. Trans. Kansas Acad. Sci., $60,1: 88,1957$. S $\mathrm{ch}$ a e f e r, H. - Studien an mitteleuropäischen Kleinsäugern, mit besonderer Berücksichtigung der Rassenbildung. Archiv f. Naturgeschichte, N. F., 4, 1: 535-590. 1935. S e t z e r, H. W. - An extra tooth in Crocidura. Jour. mammal., 38, 2: 258-259, 1957.

\section{Tadeusz BUCHALCZYK}

\section{DURCH DIPTERA AUF LEBENDEN SÄUGETIEREN GELEGTE EIER JAJA DIPTERA SKŁADANE NA ŻYWYCH SSAKACH}

Die Tatsache des Eierlegens durch Fliegen in Wunden von lebenden Tieren ist allgemein bekannt. Im Jahre 1955 beobachtete man z. B. im Sommer im Wisentreservat in Białowieża das Ausbrechen des linken Hornes mitsamt einem Schädelfragment bei dem Wisentbullen Bison bonasus ( $\mathrm{L}$ in na e u s 1758) „Poganin”, Pedigree Book. No 716, geboren 4. VII. 1947. Am nächsten Tage stellte man in der Wunde zahlreiche Larven von näher nicht bestimmten Diptera fest. Die Wunde wurde mit Erfolg mit Sulfoamiden ausgeheilt. Das Horn regenerierte sich teilweise.

Die Tatsache des Eierlegens durch Fliegen auf lebenden Igeln, Erinaceus europaeus roumanicus Barret-Hamilton 1900; beobachtete ich in Bialowieża zwei Mal. Ein junger sich in der Agonie befindender Igel wurde auf einige Stunden vor dem Tode mit Anhäufungen von Diptera-Eiern in der Region der Augen und des Maules belegt. Im Juli 1960 beobachtete ich einen kleinen Igel, der in der Ohrregion in einer Wunde Larven von näher nicht bestimmten Diptera hatte.

Auf einer am 5. VII. 1960 auf dem Areal der Białowieżaer Waldlichtung durch mich geschossenen Nyctalus noctula ( $\mathrm{S} \mathrm{ch} \mathrm{r} \mathrm{e} \mathrm{be} \mathrm{r} \mathrm{1774),} \mathrm{No.} \mathrm{coll.} \mathrm{23827,}$ fand ich durch Diptera gelegte Eier. Die geschossene Fledermaus war ein junges Weibchen (mit leicht abgeriebenen Zähnen) von 24,3 g. Körpergewicht. Die Eier der Diptera waren in der Gesamtzahl von ca 70 Stück auf zwei Stellen gruppiert und zwar auf der rechten Brust in der unmittelbaren Nähe der Zitze und 5 Eier auf der Rückenseite des Körpers in der Region des linken Schenkels. An den Stellen, wo die Eier gelegt wurden, stellte man keine Spuren irgendeiner Verletzung fest. Die Eier waren auf das Haarkleid gelegt, aber ihre Mehrzahl ganz an der Haut selbst.

Aus den gesammelten Eiern begannen am 6. VII. 1960 d. i. am folgenden Tage nach dem Tode der Fledermaus Fliegenlarven herauszuschlüpfen. Man züchtete sie auf einem Stück Schweineleber und Katzenfleisch in einem gläsernen Gefäss. Als Zuchtergebnis erhielt man am 27. VII. drei I m a g i n e s Lucilia ampulacea Vil1. und dann eine Woche später 3 Exemplare Caliphora vicina R.-D. Die Insekten wurden als solche durch A. Mońko bestimmt, wofür ich ihm an dieser Stelle herzlichst danke.

Im Lichte der oben beschriebenen Vorfälle scheint es, dass das Fallen der Körpertemperatur bei schlafenden Fledermäusen oder bei dem in der Agonie stehenden Igel, ähnlich wie auch Wunden für Fliegen ein Signal zum Eierlegen auf lebenden Tieren ist oder sein kann.

Inst. f. Säugetierforschung in Białowieża, Polnische Akad. d. Wiss. 